\title{
CELEST INESCA
}

https://doi.org/10.7203/Celestinesca.4.19508

$P R E G O N E R O$

"contarte he maravillas . . ."

CELESTINA EN LAS TABLAS: Muy recientemente, en Estados Unidos, precisamente en Tejas, se celebró el Quinto Festival de Teatro del Siglo de oro. Fue patrocinado por el National Park Service y tuvo lugar en el Chamizal National Memorial durante el periodo 7-22 de marzo de 1980. En el festival se vieron trece producciones de sendas compañias teatrales de México (con nueve grupos), de Estados Unidos (con tres: de Tejas, la Florida y Nueva York), y de España (un grupo, de Madrid). 'Obras de autores tan dis-

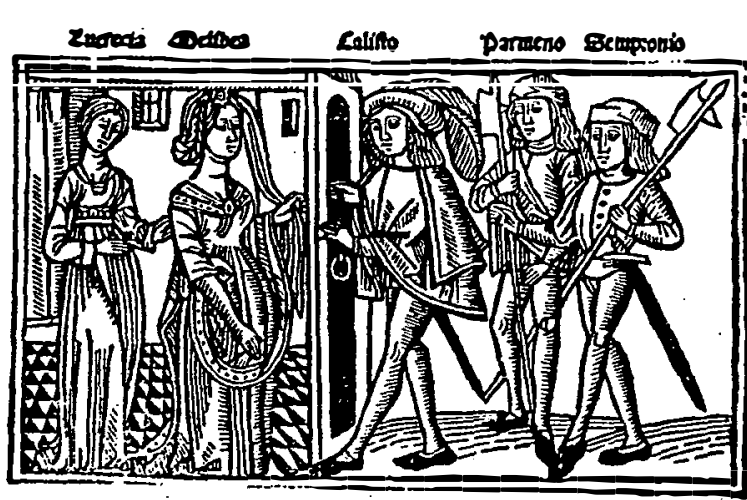

Burgos, ¿1499? tintos como son Sor Juana, Lope (2), Lope de Rueda, Ruiz de Alarcón, Valdivieliso, Calderón (3), Rojas Zoirrilla y Fernando de Rojas fueron montadas con gran éxito y aprobación de ambos los jueces y el público - . . La producción de La Celestina, que se vio dos veces el 18, era un arreglo de René Buch quien era, además, director de escena. Buch presentó una versión de $L C$ por primera vez en Nueva York en 1974 (Ver LCDB 202 y Celestinesca, 1, i [1977], pág. 35 , no. 202). Esta versión

se vio y fue premiada en el Calisto a las puertas de Melibea. Aucto XII. estreno de este mismo Festival de Chamizal (1976), lo cual explica por qué esta vez se presentó fuera de concurso. El adaptador ha ido reformando su texto original y esta versión diferia en muchos aspectos de la original, según nos informa el colega y profesor DONALD DIETZ (Texas Tech University) quien ha tenido la suerte de ver ambas producciones. Esta vez en Celestina habia OFELIA GONZALEZ; en Calisto, FRANK ROBLES y OMAR TORRES; en Melibea, YOLANDA ARENAS. . Este año hubo por primera vez un simposio después de la actuación de las representaciones compuesto de académicos selectos, actores, directores y técnicos y al cual se les invitaba a.los miembros del público. Según todas las noticias esta innovación resultó ser muy positiva. Los señores académicos que ahora participaron son el antes mencionado profesor DIETZ y: A. PEREZ PISONERO (Univ. de Tejas en El Paso), EVERETT HESSE (California State Univ. en San Diego), y HANNAH E. BERGMAN (Lehman College-CUNY) . . . . . L La compañia que dirige el señor Buch, el Teatro Repertorio Español de Nueva'York, antes de aparecer en Chamizal, llevó La Celestina a la ciudad de San Antonio donde el 15 y 16 de marzo (1980) un público muy entusiasta fue regalado con los donaires y profundidades de Rojas. Aun un poco antes, fue puesta en escena (de nuevo) en Nueva York. Esperamos poder tener una reseña de la producción 
de Chamizal en estas páginas pero por el momento, y para concretar algunos puntos sobre la calidad de esta representación, citaremos de una reseña de The New York Times (3 marzo 1980) escrita por Richard F. Shepard:

"The story smacks of Romeo and Juliet, except that it is different. It tells of the love between Calisto, a weal thy young Christian, and Melibea, the beautiful daughter of a Jewish merchant, a situation that was impermissible in that particular world. Calisto's two servants persuade him to use the cunning of Celestina, an old harridan and whore with a keen wit and a knowledge of people-handling . . . OFELIA GONZALEZ has the title role and she plays it beautifully, a woman swathed in gray robes who wheedles, laughs, fawns, rages and conspires with such skill that one never tires of watching her gestures or hearing her voice... OMAR TORRES and YOLANDA ARENAS, as the star-crossed lovers, have straight roles that they perform with much passion and utter seriousness. MATEO GOMEZ and JUAN CARLOS GIMENEZ portray the two servants with peasantlike fidelity, displaying basic emotions such as greed and lust, playfulness and passion that kills. . . the production... works on all levels, lofty and low. Even the set by ROBERT WEBER FEDERIC0, dominated by two curving staircases, helps separate the romantic higher thoughts from the baser animal instincts that appear on the stage floor."

Puede ser que la actuación fuera de esa calidad: por lo menos, es el deber del crítico reaccionar honestamente ante los actores. Pero eso de un Calisto cristiano y una Melibea judia, sacado de la's notas que acompañaban al programa y que leyó muy bien el critico Shepard, es una injusticia al texto de Rojas afirmarlo, como parte integra de su trama--y como si fuese una verdad conocida--, esta todavia muy debatida conjetura.

También en Estados Unidos. De una producción especial nos ha llegado este interesante recorte de periódico (San Francisco Chronicle, 27 julio, 1979, pảg. 60):

"Celestina, adapted from the Spanish classic of Fernando de Rojas by EDWARD SENIOR, will be presented by StageGroup Theatre at 8:30 p.m. Fridays and Saturdays, August 3 to September 22 at 449 Powell. Previews start tonight."

Estamos en contacto con una profesora que asistió con su clase a esta representación y esperamos tener para el próximo número de CELESTINESCA su comentario sobre la misma.

En Italia. Esto de mantener un tipo de simposio parece ser cada vez más común. No solo se ha hecho con la producción reciente en Chamizal, pero tuvimos ocasión de señalar simposios semejantes en nuestras noticias sobre la producción bilingüe de ALVARO CUSTODIO (en Los Angeles, 1979) y la inglesa de CHARLES LEWSEN (Sheffield, 1979). Ahora nos viene la noticia, mandada por nuestra corresponsal EMMA SCOLES (Roma), de que así ocurrió con la de ALFONSO SASTRE (estreno: Roma, 1979). La mesa redonda fue conducida por el director de escena, LUIZI SQUARZINA y "algunos hispanistas," 
después de unas semanas en el teatro Argentina (ver, para más detalles, Celestinesca 3, ii: pág. 41). Nos parece un procedimiento del todo admirable.

En España. Lo lmás nuevo en el mundo de las teatralizaciones de $L C$ será la que se montó en Madrid en el Teatro Espronceda 34 con el título Calisto y Metibea. Dirigíó ahora, como lo ha hecho durante los últimos años en varios paises de turopa, a donde ha 11 evado primero esta adaptación "libre" de RICARDO LOPE; ARANDA, el director MANUEL MANZANEQUE y su Compañia Tirso de Molina. . . El estreno tuvo lugar el 6 de febrero de 1980 y la critica, en general, ha sido severa. Ha querido el adaptador (cito sus palabras)
"recrear en el sentido de teatralizar los conceptos, traducir a 'situaciones' ciertas formas de humor pura- mente verbal en el texto; simplificar, fundiéndolos, personajes que cumplen idénticas funciones: en defini- tiva, estructurar de un modo teatral y de hoy el enorme material de la novela dialogada original, lo que conlleva, en este caso, disminuir la excesiva cantidad de muertes y ordenar sus 'tiempos dramáticos', con el fin de que co- bren mas fuerza las muertes necesarias $y$ éstas ocurran en el orden más lógico."

Las varias reacciones (ver, en este número, el s315 del suplemento bibliográfico, para las noticias de algunas de ellas) son del tipo "Ha desprovisto el tema de todo su contorno... se deja el esqueleto, se desprecia la sustancia. . . la lógica se pierde la tensión dramática se aniquila, el orden se pierde" (E. Haro Tecglen en EL Pais, 10 de febrero, 1980). - A Aparecieron en los papeles principales, en buenas actuaciones, MARIA GUERRERO (Celestina), INMA DE SANTY (Mel ibea), y JOSE SANCHO (Calisto). En los otros papeles había: ARACELY CONDE, MARIA VIDAL, GONZALO SANMIGUEL, RAMON PONS y JOSE MARIA ESCUER.

Recientemente. . $\propto$

LC EN TRADUCCION. - . nuestra corresponsal de Hungria, la profesora KATALIN KULIN nos pone esta noticia sobre la nueva traducción al húngaro de la obra de Rojas. Es de fecha 1979. SANDOR KAROLY tradujo la Comedia que, ahora en traducción refundida y extendida a la Tragicomedia por FERENC SZJNYI, incluso todas las materias pre- y posliminares, merece elogios de la profesora KULIN, ella misma autora de un inteligente posfacio.

LC EN LOS CONGRESOS .. . En las sesiones de la Midwest MLA, convocadas en Indianapolis, USA. en noviembre de 1979, fue leída la ponencia de ARTHUR C. OLDS (Michigan State Univ.), "Structure and Narrative Technique in $L C$ : The Asides." El mismo nos informa que acabará en breve su tesis doctoral sobre unos aspectos de la ironia en $L C$. Su ponencia fue seguida por los comentarios del profesor E. J. WEBBER (Northwestern U.) y la sesión fue presidida por otro buen conocedor del texto rojano, JERRY R. RANK (U. of Illinois - Chicago Circle). . . En las sesiones celebradas 
en mayo en Kalamazoo, Michigan, de la Medieval Conference, ADRIENNE S. MANDEL (California State $U$. en Northridge) presidió una reunión especial sobre el tema del debate (anti) feminista del siglo XV. En ella participaron los celestinistas COLBERT NEPAUL-SINGH (SUNY-ATbany), ANTONY VAN BEYSTERVELDT (Bowling Green State U.) y JAMES R. STAMM (New York Univ.). El último de los tres pronunció un discurso con especial relevancia para nosotros, "LC: The Debate Ends." Su idea central es que LC es la última obra de relieve en que dicho debate es empleado como elemento estructural (hasta no La Galatea de Cervantes). A fin de cuentas, hasta Rojas lo rechaza a favor de una actitud realista de "gaudeamus" o de "carpe diem."

Agradecemos a JOSE LUIS CANO la noticia sobre Celestinesca aparecida en Insula, núms. 396-397 (nov.-dic. 1979), en la página 2. Nuestro correo nos demuestra la gran utilidad de la gentileza que nos ha hecho.

Hacia el futuro...

Una labor bibliográfica se va terminando.. . La bibliografía de las ediciones antiguas de $L C$ que desde hace tiempo vienen preparando las profesoras EMMA SCOLES (Roma) y ERNA BERNDT KELLEY (Smith College) en colaboración con dos alumnas en Italia parece estar en sus últimas reformaciones. Será de mucha importancia cuando aparezca; la necesidad de tener claramente expuesta la dificultosa huella dejada en el mundo de la imprenta por la obra de Rojas ahora encuentra respuesta.

El tema de las menciones de animales en $L C$ será estudiado en una tesis de licenciatura que actualmente se prepara en Roma . . .

Un articulo destinado a las paǵinas de Hispania (USA) es uno de DAVID WISE (Texas Women's Univ.), "Reflections of Andreas Capellanus's 'De reprobatio amoris' in Juan Ruiz, Alfonso Martinez de Toledo, and Fernando de Rojas." - . - Selecciones Austral (Espasa-Calpe) anuncia la inminente aparición de su texto de $L C$ con un prólogo de PEDRO M. PIÑEIRO . . . Está preparando una monografía sobre tema celestinesco FERNANDO DE TOROGARLAND (Madrid) que será acompañada de una bibliografía completa de la critica, ediciones, etc.

Otras cosas en preparación incluyen una edición crítica de la Tragicomedia por el profesor MIGUEL MARCIALES (Venezuela) y una monografia de titulo alentador: La comedia humanistica en LC: dos muestras prospectivas de un género literario. Esta saldrá en la serie "Theses et Studia Scholastica" patrocinada por el Seminario de Filologia Hispánica (Santiago de Chile) y dirigida por el profesor MARIO FERRECIO PODESTA. Estas actividades de los colegas sudamericanos demuestran la salud de $L C$ en todas partes del mundo de habla española.

LOUISE FOTHERGILL-PAYNE (Univ. of Calgary) publicará un artículo en cuadernos hispanoomericonos (1981 o 1982) sobre algunas rarezas del siglo XVI en el ámbito de las adaptaciones dramáticas. El titulo: "La cambiante faz de la Celestina." . . . La misma investigadora ha topado con unas traducciones del siglo XV de Séneca que bien podrian ser una fuente 


\section{CELESTINESCA}

primaria de la Comedia. Ha presentado, en forma de charlas, algunas de estas materias ya en Nottingham, Londres, Toronto y Montreal. Mientras tanto procede con un estudio monográfico de estas nuevas influencias senequistas.

Habrá definitivamente una sesión especial dedicada a $L C$ en el congreso de la MLA (Houston) en diciembre de 1980. Actualmente está organizándola la profesora MANDEL. Se anunciará en breve el programa y los participantes. . En el VII Congreso de la Asociación Internacional de Hispanistas, tercera circular, abril de 1980, se anuncia un solo trabajo sobre $L C$, JOSEPH SNOW (Georgia), "Un aspecto del arte teatral de Celestina: el caso de Claudina."

Se cierra esta edición del PREGONERO con gracias a las personas que proporcionaron informes de todo tipo: Robert BLACK, Geoffrey WEST, KarlLudwig SELIG, A. CUSTODIO, J. C. TEMPRANO, Donald DIETZ, E. SCOLES, S. WHITTAKER, K. KISH, G. SIEBENMANN, M. FERRECIO, J. R. STAMM, L. NORDENHOLZ, L. FOTHERGILL-PAYNE y K. KULIN.<smiles>CC(=O)OCCCCCO</smiles>

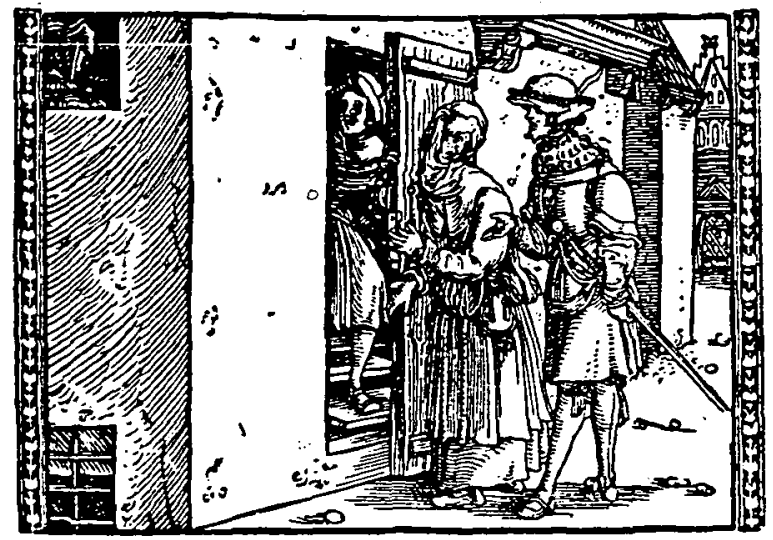

Celestina y Sempronio llegan a la casa de Calisto. Aucto V. De la traducción alemana de C. Wirsung (1520). 


\section{TEATro Esproncedo 34}

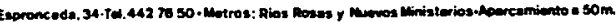

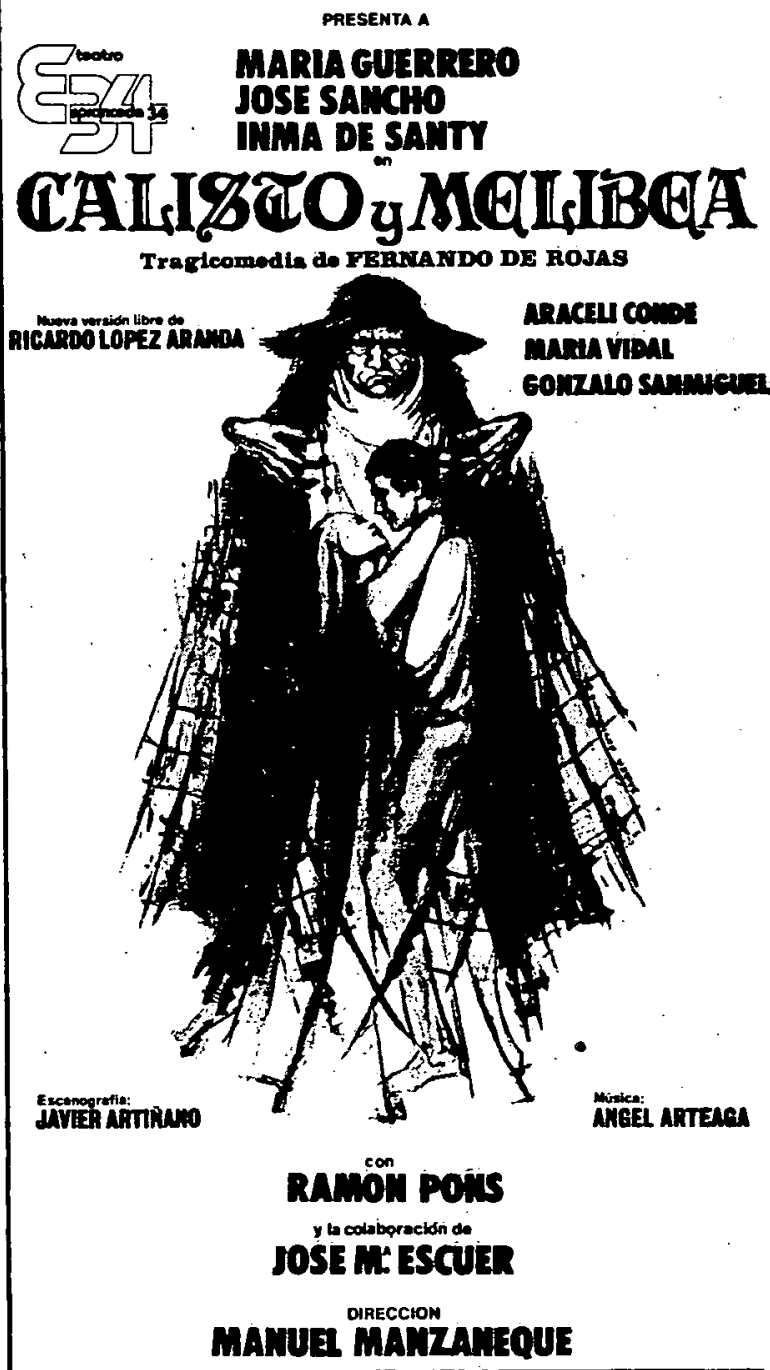

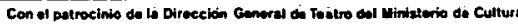

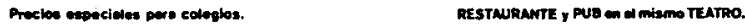

Reproducción de la cubierta del programa de una teatralización de Calisto y Melibea, en Madrid. 